\title{
Barriers and facilitators in implementing a pilot, pragmatic, telemedicine-delivered healthy lifestyle program for obesity management in a rural, academic obesity clinic
}

John A. Batsis ${ }^{1 *} \mathbb{D}$, Auden C. McClure ${ }^{2,3}$, Aaron B. Weintraub ${ }^{3}$, Diane Sette ${ }^{3}$, Sivan Rotenberg ${ }^{3,4}$, Courtney J. Stevens 3,4, $^{3,}$ Diane Gilbert-Diamond ${ }^{2,3,6}$, David F. Kotz ${ }^{7}$, Stephen J. Bartels ${ }^{8}$, Summer B. Cook ${ }^{9}$ and Richard I. Rothstein ${ }^{2,3}$

\begin{abstract}
Purpose: Few evidence-based strategies are specifically tailored for disparity populations such as rural adults. Twoway video-conferencing using telemedicine can potentially surmount geographic barriers that impede participation in high-intensity treatment programs offering frequent visits to clinic facilities. We aimed to understand barriers and facilitators of implementing a telemedicine-delivered tertiary-care, rural academic weight-loss program for the management of obesity.

Methods: A single-arm study of a 16-week, weight-loss pilot evaluated barriers and facilitators to program participation and exploratory measures of program adoption and staff confidence in implementation and intervention delivery. A program was delivered using video-conferencing within an existing clinical infrastructure. Elements of Consolidated Framework for Implementation Research (CFIR) provided a basis for assessing intervention characteristics, inner and outer settings, and individual characteristics using surveys and semi-structured interviews. We evaluated elements of the RE-AIM model (reach, adoption) to assess staff barriers to success for future scalability.
\end{abstract}

Findings: There were 27 patients and 8 staff completing measures. Using CFIR, the intervention was valuable from a patient participant standpoint; staff equally had positive feelings about using telemedicine as useful for patient care. The RE-AIM framework demonstrated limited reach but willingness to adopt was above average. A significant barrier limiting sustainability was physical space for intervention delivery and privacy and dedicated resources for staff. Scheduling stressors were also a challenge in its implementation.

(Continued on next page)

\footnotetext{
* Correspondence: john.batsis@gmail.com

Work was performed while at Dartmouth-Hitchcock.

'Division of Geriatric Medicine, Department of Nutrition, University of North

Carolina at Chapel Hill, Chapel Hill, NC, USA

Full list of author information is available at the end of the article
}

(c) The Author(s). 2020 Open Access This article is licensed under a Creative Commons Attribution 4.0 International License, which permits use, sharing, adaptation, distribution and reproduction in any medium or format, as long as you give appropriate credit to the original author(s) and the source, provide a link to the Creative Commons licence, and indicate if changes were made. The images or other third party material in this article are included in the article's Creative Commons licence, unless indicated otherwise in a credit line to the material. If material is not included in the article's Creative Commons licence and your intended use is not permitted by statutory regulation or exceeds the permitted use, you will need to obtain permission directly from the copyright holder. To view a copy of this licence, visit http://creativecommons.org/licenses/by/4.0/ The Creative Commons Public Domain Dedication waiver (http://creativecommons.org/publicdomain/zero/1.0/) applies to the data made available in this article, unless otherwise stated in a credit line to the data. 
(Continued from previous page)

Conclusions: The need to engage staff, enhance organizational culture, and increase reach are major factors for rural health obesity clinics to enhance sustainability of using telemedicine for the management of obesity.

Trial registration: Clinicaltrials.gov NCT03309787. Registered on 16 October 2017.

Keywords: Obesity, Telemedicine, Rural, Weight loss, Pragmatic

\section{Contributions to the literature}

- Technology-delivered care can be helpful in scaling interventions for rural adults with obesity residing in remote areas

- Policymakers can enhance reimbursement and incentives to promote telemedicine-delivered care to adults

- Enhancing patient/staff engagement and culture can overcome organizational stress and enhance reach of the intervention

\section{Background}

Tertiary care weight management clinics located at academic medical centers usually serve patients from large geographic areas. Travel becomes a significant service barrier to adults residing in remote and isolated areas, and hence, disorders such as obesity may not be perceived by patients as urgent or harmful [1]. Routinely integrating telemedicine into clinical infrastructures may provide an opportunity to address these hurdles. Telemedicine can increase the exposure for low-resource populations without access to specialized services, particularly in conditions that do not necessarily require physical touch.

Previous health behavior change interventions using telemedicine effectively improved readiness to change [2] and provided effective psychiatric treatment [3]. Recognizing the need for different models of health service delivery, we conducted a pragmatic clinical pilot trial within our weight-management clinic aimed at providing usual clinical care using two-way, live, telemedicine. Our results were proven to be feasible, acceptable, and potentially effective at producing weight loss [4]; yet, this delivery system (telemedicine) could potentially lead to inherent challenges for both patient and provider stakeholders. Successful implementation of evidence-based practices requires an understanding of the barriers and facilitators of implementing new services. Two wellrecognized frameworks for exploring implementation factors, barriers/enablers that permit planning, evaluating practice change interventions, and why implementation succeeded or not to identify modifiable factors, are the Consolidated Framework for Implementation Research (CFIR) and RE-AIM [5]. These frameworks have previously been applied in the context of telemedicine and have found that patient and leadership engagement, comfort levels with the technology, and a need to have similar efficacy and workflows to in-person visits were important factors [6-8]. As such, the purpose of this analysis was to apply these established implementation frameworks to refine our intervention for a future, large-scale trial that could ensure its long-term success and program sustainability in rural areas. We anticipate that our findings could provide useful guidance to others in implementing evidence-based practices.

\section{Methods}

\section{Study setting}

Dartmouth-Hitchcock (D-H) is a 396-bed hospital affiliated with Dartmouth College, an Ivy League University, and is New Hampshire's only teaching hospital. Ambulatory services provide care for 1.5 million patients yearly, consisting of 1.3 million outpatient visits. This rural medical center is located in Lebanon, New Hampshire, USA, a small town of 13,522 persons, within Grafton County (population 89,396) [9], adjacent to the Vermont border in the northeastern USA. The Center for Telehealth leveraged institutional resources to assist with setup, deployment, and use of telemedicine throughout the study and was responsible for orientation, training, and setup of the software; hardware requirements; configuration of the tablets; and support, with troubleshooting back-up provided by the research assistant for any difficulties.

\section{Intervention study design and description}

The clinical trial was conducted between December 2017 and September 2018 within the Weight \& Wellness Center clinic. During this time, the clinic saw 385 new consultations for adult obesity management. The study was approved by the Committee for the Protection of Human Subjects and the Dartmouth-Hitchcock Institutional Review Board and was registered at clinicaltrials. gov \#NCT03309787. The trial was a single-arm, evidence-based weight-loss intervention [10] consisting of an individualized 1:1 exercise and nutrition counseling behavioral change program. Participants were 
referred by their primary care provider. We enrolled 37 participants, of which 27 completed the intervention. Participants were approached by the on-site clinician, and if interested, a research assistant obtained informed consent. Study recruitment methods have previously been described [4].

The 16-week Healthy Lifestyle Program was based on the Diabetes Prevention Program [11] focusing on health-behavior change delivered using individual, 1:1 sessions with trained staff (see below) using telemedicine. Patients underwent a comprehensive multidisciplinary intake. Visits lasted $30 \mathrm{~min}$ each, conducted by the interventionists in the clinic using videoconferencing. Each used motivational interviewing, goal-setting, and coaching strategies for the telemedicine participants to parallel the on-site program being delivered for clinical care. Supplemental File \#1 presents the specific curriculum. We previously demonstrated that this program was acceptable and effective in delivering the in-person program using telemedicine on weight loss [4].

\section{Intervention implementation Study design}

The aim of this current analysis and manuscript was to provide information of the telemedicine-delivered intervention in the pre-implementation phase that could inform a future, large-scale intervention and could provide useful information to other rural obesity practices. For this component of the study, study participants and staff completed questionnaire measures (see below) and a semi-structured exit interview. The Standards for Reporting Implementation Studies are in supplemental file \#2 [12].

\section{Study participants}

Study sessions were offered by a health coach, nurse exercise specialist, and registered dietitian each of whom were licensed and trained, having experience working with patients with obesity for at least 5 years. An administrative assistant and a clinical secretary were responsible for scheduling patient participants and was conducted as per usual clinical care guidelines and provider schedules. A practice manager oversaw the clinical operations and managed staff within the clinic. Two physicians and an advanced practice registered nurse informed prospective participants of the study during their initial, in-person consultations. A research assistant was responsible for all project management tasks, including troubleshooting of the encounters, data entry and management, and analysis. There were 27 study completers and 8 staff who completed postintervention follow-up survey measures and an exit interview on the barriers and facilitators of the intervention. Interviews were all conducted by the lead author (JAB) and were structured using the above frameworks. Questions posed to staff are presented in Supplementary File \#3 and those to patient participants in Supplementary File \#4.

\section{Implementation assessment frameworks}

We explored barriers and facilitators, including elements from the Consolidated Framework for Implementation Research (CFIR) [13] and the RE-AIM (Reach, Effectiveness, Adoption, Implementation, Maintenance) [14] frameworks.

\section{CFIR outcome measures}

We evaluated the intervention characteristics using a satisfaction questionnaire (Table 1) to patient participants (1-5 Likert scale, low to high). To measure the inner setting, staff $(n=8)$ completed a 38-measure questionnaire [15] that evaluated culture, culture stress, culture effort, implementation climate, learning climate, leadership engagement, and available resources (Table 2, Supplemental File \#4). We assessed organizational change using an adapted version (Supplemental File \#5) of the General Organizational Index [16] administered to staff. This scale is an interview of 11 domains with a 5-point rating of program philosophy, commitment, client eligibility and identification, health promotion plan and its treatment, training, process and outcome monitoring, assessment, quality assurance, choice supervision, and penetration. The Outer setting and Characteristics of the Individuals involved single questions (range 1-10) evaluating whether staff had confidence in the intervention

Table 1 Intervention characteristics - patient perspective of ease of use and value of technology $(n=27)$

\begin{tabular}{|c|c|c|c|}
\hline & Mean $\pm S D^{a}$ & Median & Range \\
\hline \multicolumn{4}{|l|}{ Ease of use of technology } \\
\hline Did you feel that the overall intervention was beneficial and worth your time? & $4.6 \pm 0.7$ & 5 & $2-5$ \\
\hline How would you rate your level of satisfaction with the video-conferencing device? & $4.4 \pm 0.8$ & 5 & $2-5$ \\
\hline How helpful was video-conferencing in assisting you to achieve your goals? & $4.4 \pm 0.7$ & 4 & $2-5$ \\
\hline Did you find the video-conferencing easy to use without much difficulty? & $4.9 \pm 0.3$ & 5 & $4-5$ \\
\hline
\end{tabular}


Table 2 Staff inner setting evaluation using Fernandez evaluation $(n=8)[15]$

\begin{tabular}{|c|c|c|c|c|c|}
\hline Subscale & \# Questions & Mean & Median & Range & Maximum survey score \\
\hline Organizational culture & 9 questions & $39.9 \pm 3.2$ & 40 & $36-44$ & 45 \\
\hline Culture stress & 4 questions & $12.3 \pm 2.7$ & 12.5 & $8-16$ & 20 \\
\hline Culture effort & 5 questions & $23.5 \pm 1.5$ & 23.5 & $21-25$ & 25 \\
\hline Implementation climate & 4 questions & $14.1 \pm 2.0$ & 14 & $11-18$ & 20 \\
\hline Learning climate & 5 questions & $21.3 \pm 3.3$ & 20 & $17-25$ & 25 \\
\hline Leadership engagement & 4 questions & $13.5 \pm 2.1$ & 14 & $10-16$ & 20 \\
\hline Available resources & 7 questions & $22.1 \pm 2.2$ & 22 & $20-27$ & 35 \\
\hline Total & 38 questions & $146.6 \pm 8.6$ & 143.5 & $139-163$ & 190 \\
\hline
\end{tabular}

Mean \pm standard deviation, median, and range are listed for the measures. The last column represents the maximum score for each subscale. This is a 38 item measure evaluating culture, culture stress, culture effort, implementation climate, learning climate, leadership engagement, and available resources. Supplemental File \#3 outlines each subscale's detailed questions and scoring from each component

delivery (Table 3). Interviews explored the perceived advantages, disadvantages, and value of the pilot both to patient participants and staff, the latter previously published [4]. We qualitatively assessed staff barriers to success for future scalability (Table 4).

\section{RE-AIM assessment measures}

Using RE-AIM, we estimated Reach, which consisted of the proportion of the number enrolled to the number evaluated for consultation during this time. Haug's staff Adoption questionnaire (Table 5) [68] permitted quantitative evaluation of the stage of change, experience, attitudes, organizational barriers, and strategies to support evidence-based practices (Likert 1-5). Perceived staff workflow delays were also evaluated (Likert 1-10 scale). Qualitative interviews inquired about the patient and staff's experience with the intervention; for staff, we explored whether it enhanced or interfered with workflow, could be sustainable, or if there were other technical or other difficulties in delivering care.

\section{Data analysis}

Descriptive statistics were computed for continuous variables; for single item questions, proportions (where appropriate) were calculated. All interviews were digitally recorded in duplicate and transcribed. Data were aggregated into Dedoose [Hermosa Beach, CA]. Transcripts were read (JAB) who conducted open coding, with themes independently verified by $A B W$, an approach that enhances research rigor by allowing for different viewpoints [18]. A codebook was developed using researcher-driven codes derived from each interview and codes generated through an inductive review of the transcripts. Focused coding using themes identified during open coding permitted defined analyses through data immersion. A query tool retrieved the text by code, reviewed for content, relevance, and prevalence of themes and grouped. Themes and comments were mapped to specifically to barriers/facilitators of using telemedicine, including its impact on clinical care using pre-specified questions related to the specific elements of the framework. The main themes reported in this study were identified, reported, and aligned, where possible to CFIR/RE-AIM.

\section{Results}

\section{Patient cohort}

Of the 27 who completed $>75 \%$ of all sessions, mean participant age was $46.1 \pm 12.3$ years $(88.9 \%$ female $)$ with a body mass index of $41.3 \pm 7.1 \mathrm{~kg} / \mathrm{m}^{2}$. Mean weight loss was $2.22 \pm 3.18 \mathrm{~kg}(2.1 \%$ change; $p<0.001)$. There was a loss in waist circumference of $3.4 \%(p<0.001)$ [4]. The mean distance from the participant's home to the center was $38.8 \pm 31.6$ miles (mean driving time $36.0 \pm 29.0$ min). Table 1 describes the intervention characteristics from the patient perspective, including the benefit of telemedicine (4.9/5), its value and ease of use (4.6/5), and its satisfaction and helpfulness (4.4/5). All were

Table 3 Staff confidence in the intervention delivery $(n=8)$

\begin{tabular}{|c|c|c|c|}
\hline & Mean \pm SD & Median & Range \\
\hline My "buy-in" was very high for this project & $6.9 \pm 3.0$ & 8.0 & $3-10$ \\
\hline I promoted the Telehealth Project to Patients & $7.3 \pm 2.8$ & 8.0 & $2-10$ \\
\hline I think that Telehealth can improve care quality & $7.6 \pm 3.1$ & 8.5 & $1-10$ \\
\hline I think the clinic is ready to adopt telemedicine in one form or another & $7.3 \pm 3.1$ & 8.5 & $3-10$ \\
\hline I value telemedicine as an emerging technology & $9.1 \pm 1.1$ & 9.5 & $7-10$ \\
\hline
\end{tabular}

Scores range from 1-10 to low to high 


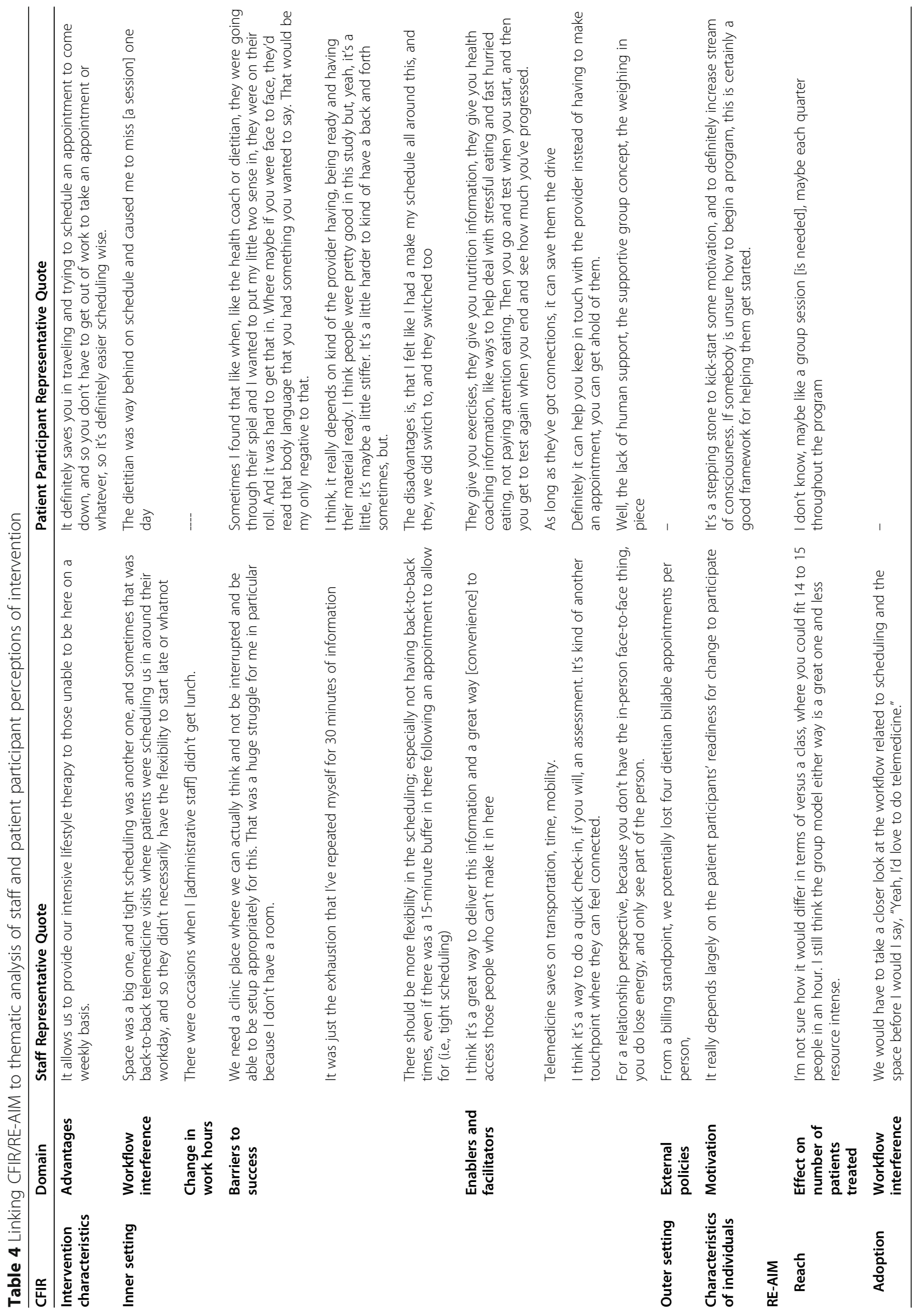


Table 5 Staff adoption questionnaire [17] $(n=8)$

\begin{tabular}{|c|c|c|}
\hline & Mean \pm SD & Range \\
\hline \multicolumn{3}{|l|}{ Staff adoption questionnaire } \\
\hline \multicolumn{3}{|l|}{ Attitudes-positive outcome } \\
\hline Using a treatment manual helps a therapist to evaluate and improve his or her clinical skills ${ }^{a}$ & $3.6 \pm 0.5$ & $3-4$ \\
\hline $\begin{array}{l}\text { Following a treatment manual will enhance therapeutic outcomes by insuring that the treatment being used is supported } \\
\text { by research }\end{array}$ & $3.4 \pm 0.7$ & $2-4$ \\
\hline $\begin{array}{l}\text { If a treatment has been shown scientifically to be effective, then the counselor is ethically obligated to use the treatment } \\
\text { as opposed to one that has not been studied }\end{array}$ & $3.3 \pm 1.0$ & $2-5$ \\
\hline \multicolumn{3}{|l|}{ Attitudes-negative process } \\
\hline Evidence-based practices make counselors more like technicians than caring human beings & $3.6 \pm 1.2$ & $2-5$ \\
\hline Treatment manuals are appropriate for research clients but not "real world" clients & $3.5 \pm 0.9$ & $2-5$ \\
\hline Using evidence-based practices detracts from the authenticity of the therapist interaction & $4.0 \pm 0.5$ & $3-5$ \\
\hline \multicolumn{3}{|l|}{ Organizational barriers } \\
\hline Evidence-based practices seem overly complicated and hard to put into practice & $3.8 \pm 0.9$ & $2-5$ \\
\hline There are influential clinicians at my program that are definitely against evidence-based treatments. & $4.4 \pm 0.9$ & $3-5$ \\
\hline $\begin{array}{l}\text { It would take some very strong incentives, such as restricting our funding, before our treatment program would use } \\
\text { evidence-based practices }\end{array}$ & $4.3 \pm 0.9$ & $3-5$ \\
\hline $\begin{array}{l}\text { The idea of evidence-based practices sound good in "theory," but in reality, it is virtually impossible to scientifically test a } \\
\text { phenomenon as complex as substance abuse treatment }\end{array}$ & $3.9 \pm 1.2$ & $1-5$ \\
\hline The treatments that we do at our program may not be "evidence-based," but they work just as well, or better. & $3.4 \pm 1.2$ & $2-5$ \\
\hline $\begin{array}{l}\text { As long as they do not conflict with treatments already in place at our program, I do not see any problem with using a } \\
\text { few procedures that are evidence-based }{ }^{a}\end{array}$ & $4.0 \pm 0.5$ & $3-5$ \\
\hline
\end{tabular}

1 strongly disagree; 5 strongly agree

SD standard deviation

${ }^{\mathrm{a}}$ Reverse score

strongly favorable to patients. Patient participants had similar favorable sentiments regarding telemedicine as did staff (see below).

\section{Implementation measures}

Data on the Inner Setting Evaluation is presented in Table 2 (Supplemental File \#5). Both culture stress and available resources were challenging, while organization culture and effort to implement was strongly favorable. The adapted General Organizational Index [16] demonstrated two concerns: client identification and process monitoring. Staff confidence in intervention delivery is presented in Table 3. Generally, staff had positive feelings regarding using telemedicine and noted above average expectations regarding its usefulness (Table 4). Haug's staff adoption questionnaire [17] is presented in Table 5. Results suggest an average to above-average willingness for staff to adopt telemedicine. While the range of answers were broad, the median response score was high, suggesting that staff felt confident that this intervention had considerable potential.

Table 5 represents the major domains and themes that emerged using select elements from the CFIR and RE-AIM frameworks. Representative quotes are presented including the usefulness of telemedicine, its advantages, and the loss of value that may occur using this technology. Space for conducting videoconferencing was a major barrier to success. Many believed that a hybrid intervention (part in-person, part remote) might reduce depersonalization. Dedicated resources from senior administration were needed to ensure successful delivery of telemedicine within the clinical framework.

Using RE-AIM, we evaluated reach and adoption. We estimated that this program only reached 37 participants with telemedicine (e.g., enrolled participants) of a potential 385 participants (9.6\%) that needed treatment for obesity. Some crucial findings on workflow interference, the impact on reach, work hours, and barriers to success are also presented. Staff felt that the study did lead to workflow delays (mean 5.0 \pm 1.7 , median 5.0, range 2-7), but few believed their existing tasks were altered considerably $(3.5 \pm 3.4$, median 1.5, range 1-9).

\section{Discussion}

The importance of engaging patient and provider stakeholders in effectively delivering telemedicine within a clinical setting cannot be overstated. Our results highlight the characteristics needed for clinical execution prior to full-scale deployment in order to maximize scalability and dissemination. There was clear value to 
patient participants with minimal challenges of using telemedicine.

Staff faced several workflow delays as evidenced by our mixed-methods assessment. Workspace issues impacted all domains. While space is limited in many healthcare centers [19], telemedicine requires a dedicated infrastructure that includes a workspace free from distractions to effectively engage in the two-way conversation and preserve privacy. In our environment, health coaches are situated in a large workroom and hence video-delivery may lead to additional distractions beyond privacy concerns. The findings observed in this study parallel those observed by Brown who found that primary-care providers voiced concerns that staffing, space, and aligning the sessions on days/times when staff/patients were available [20] were barriers to telemedicine implementation. Our data suggest that staff engagement was highly reliant on the ability to adequately conduct and schedule such encounters. Whether specific computer peripherals can be used (i.e., noise-canceling headphones) is a possibility. The inability to integrate sessions within routine in-person care was a major impediment to success and highly dependent on several external factors. Providers are constrained in their ability to run on time based on rooming, scheduling, and patient arrival times that could lead to downstream delays. Increasing visit times or providing breaks between telemedicine sessions and in-person sessions may be a potential solution.

"Inner setting" factors impacting staff satisfaction included participants characteristics. All screened patients were offered the intervention. Readiness to change is a known determinant of attrition and intervention compliance for obesity treatment programs [21]. Our program did not screen participants on the basis of this measure, leading to participants who may have been less motivated to initiate change being included in the sample. Staff felt that 1:1 visits were not cost-effective and that group-based therapy would enhance provider satisfaction. Operationally group visits could improve downstream revenues. Such a hybrid in-person/remote program also adds the benefit of in-person touches and social connectedness that may not be observed with full-remotely delivered care. Generally, staff had confidence in this mode of delivery, although adopting this intervention without future changes would be problematic. These factors have previously been observed with the participants completing this intervention [4].

The evidence-based Veterans Affairs MOVE program [22, 23] and their telemedicine results have demonstrated favorable effectiveness outcomes [24]. Our preliminary results suggested similar challenges including the need for more staff time. A randomized trial testing a weight-loss intervention delivered via telehealth for rural cardiac rehabilitation patients (mean age $63 \pm 9.3$ years) also demonstrated usefulness and feasibility, but studies are markedly underpowered. Additional research is needed to further this modality with such populations [25].

Implementing a rigorous evaluation could enhance, scale, and expedite dissemination of research-level programs as evidenced by the TeleMOVE program [26]. Sustainability is most important, both operationally and financially. Without such information, it would be difficult to modify programs to permit longterm solvency. Additionally, our intervention was implemented in a culture conducive to process change. There was also engagement of the academic missions from senior stakeholders. Our investigation also had a number of limitations. First, our pilot was nonrandomized and limited to eight personnel providing information on clinic-related implementation outcomes. While we acknowledge that it would be hard to implement a large-scale project using data from this small number of staff, it does provide the team with important information to conduct a larger, type I hybrid, effectiveness-implementation trial. Second, our results acknowledge the contextual and organizational factors that could impact future implementation. Third, we did not formally evaluate the fidelity of the intervention or other measures of implementation that could potentially foster longterm sustainability. Last, while the intervention provided partial support to a research coordinator, none of the clinical staff were reimbursed, in line with a pragmatic intervention. Whether other centers are similarly supported is unknown.

\section{Implementation implications for other rural health practices}

While exploratory, our implementation outcomes provide useful information to scale-up and spread innovative healthcare interventions [27]. By incorporating our suggestions to address shortcomings, we can promote sustainability, success and improved patient outcomes [28-30]. We recognize that not all elements of CFIR or RE-AIM were evaluated and that this may limit our ability to effectively integrate specific elements that can be helpful in long-term dissemination. However, our experience provides formative information for others to consider in implementing telemedicine in other venues, including primary and specialty care using the abovenoted frameworks:

a. Disseminating an evidence-based intervention: This exploratory data demonstrates that the 
intervention characteristics (CFIR) from a provider and patient participant standpoint were favorable suggesting that the Healthy Lifestyle Program can be scaled favorably to other centers. Importantly, our results suggested that staff had favorable attitudes and confidence in adopting the intervention (RE-AIM).

b. Patient/staff engagement: The lack of a face-toface encounters led to depersonalization, suggesting a need and value of periodic in-person visits for patient. Rather than the entire intervention be conducted remotely, a hybrid in-person/remotely delivered program could promote social engagement and connectedness among participants, but also among staff (RE-AIM). However, only a future trial could best test this delivery strategy.

c. Enhancing organizational culture: Our experience noted that mixing telemedicine visits with on-site visits led to consider stress and inefficiencies amongst staff (CFIR). Block scheduling (e.g., all telemedicine visits occurring in sequence) could easily overcome these challenges.

d. Overcoming organizational stress: Key barriers to successful implementation of telemedicine-based interventions include the need for a dedicated space for telemedicine to eliminate potential distractions. Our experience suggested that conducting telemedicine in large workspaces leads to distractions and considerable stress to study staff (CFIR).

e. Increasing reach: Current 1:1 sessions reduce the potential reach of participants and should be reserved for those needing personalized therapy (RE-AIM). Group remotely delivered sessions can reduce staff burden and fatigue but increase the ability to reach more people with limited resources.

\section{Conclusions}

Applying implementation evaluation frameworks such as CFIR and RE-AIM in our pragmatic pilot intervention of a telemedicine-delivered wellness intervention provided data in our pre-implementation phase that could inform future trial sustainability and spread not only locally, but to other centers and other specialties. Our approach is novel in that it incorporates elements of implementation science in a rural academic infrastructure that, to our knowledge, has not been fully explored that could be helpful to enhance obesity care within a specialty, rural care environment to provide data on how to maximize the impact of practice-based interventions.

\section{Supplementary information}

Supplementary information accompanies this paper at https://doi.org/10. 1186/s43058-020-00075-9.

Additional file 1: Supplemental File 1: Components of the Healthy Lifestyle Program at the Dartmouth Weight and Wellness Center. Supplemental File 2 Standards for Reporting Implementation Studies: the StaRl checklist for completion. Supplemental File 3 - Staff Questions. Supplementary File 4 - Patient Satisfaction Questions. Supplemental File 4: Inner Setting Measures from the CFIR Framework - Fernandez et al (1low to 5-high, strongly disagree to strongly agree): $(n=8)$. Supplemental File 5 - Adapted General Organizational Index.

\section{Abbreviations}

CFIR: Consolidated Framework for Implementation Research; DH: Dartmouth-Hitchcock; RE-AIM: Reach, Effectiveness, Adoption, Implementation, Maintenance

\section{Acknowledgements}

We thank the Center for Telehealth (Mary Lowry, Vanessa Brown, Fredric Glazer) for their assistance in developing the telemedicine component, and Tara Efstathiou, Laurie Gelb, Eugene Soboleski, Jane Brewer, Martha Catalona, Philip Oman, and Kaitlyn Christian, for their administrative assistance at the Weight and Wellness Center.

\section{Authors' contributions}

JAB — design, conception, analysis, interpretation, approval of the final version. ACM-design, conception, analysis, interpretation, approval of the final version. ABW - design, conception, analysis, interpretation, approval of the final version. DS - analysis, interpretation, approval of the final version. SR-design, conception, analysis, interpretation, approval of the final version. CJS-design, conception, analysis, interpretation, approval of the final version. DGB-design, conception, analysis, interpretation, approval of the final version. DFK-design, conception, analysis, interpretation, approval of the final version. SJB-design, conception, analysis, interpretation, approval of the final version. SBC-design, conception, analysis, interpretation, approval of the final version. RIR-design, conception, analysis, interpretation, approval of the final version.

\section{Funding \\ Dr. Batsis receives funding from the National Institute on Aging of the National Institutes of Health under Award Number K23AG051681 and from the Friends of the Norris Cotton Cancer Center at Dartmouth and National Cancer Institute Cancer Center Support Grant 5P30 CA023108-37 Develop- mental Funds. Dr. Batsis also receives funding from the Patient Centered Ori- ented Research Institute. Dr. Batsis has also received honoraria from the Royal College of Physicians of Ireland, Endocrine Society, and Dinse, Knapp, MCAndrew LLC, legal firm. This study was funded by The Dartmouth Clinical and Translational Science Institute, under award number UL1TR001086 from the National Center for Advancing Translational Sciences (NCATS) of the Na- tional Institutes of Health (NIH). Support was also provided by the Dartmouth Health Promotion and Disease Prevention Research Center supported by Co- operative Agreement Number U48DP005018 from the Centers for Disease Control and Prevention.}

\section{Availability of data and materials}

The datasets generated and/or analyzed during the current study are not publicly available due to data privacy policies at Dartmouth-Hitchcock but may be available from the corresponding author on reasonable request.

\section{Ethics approval and consent to participate}

The study was approved by the Committee for the Protection of Human Subjects at Dartmouth College and registered at clinicaltrials.gov \# NCT03309787.

\section{Consent for publication}

N/A 


\section{Competing interests}

There are no competing interests. There are no conflicts of interest pertaining to this manuscript.

\section{Author details}

${ }^{1}$ Division of Geriatric Medicine, Department of Nutrition, University of North Carolina at Chapel Hill, Chapel Hill, NC, USA. ${ }^{2}$ Geisel School of Medicine at Dartmouth and The Dartmouth Institute for Health Policy \& Clinical Practice, Lebanon, NH, USA. ${ }^{3}$ Dartmouth Weight \& Wellness Center, Lebanon, NH, USA. ${ }^{4}$ Department of Psychiatry, Dartmouth-Hitchcock, Lebanon, NH, USA. ${ }^{5}$ Dartmouth Centers for Health and Aging, Dartmouth College, Hanover, $\mathrm{NH}$, USA. ${ }^{6}$ Department of Epidemiology, Dartmouth College, Hanover, NH, USA. 'Department of Computer Science, Dartmouth College, Hanover, NH, USA. ${ }^{8}$ Mongan Institute, Massachusetts General Hospital, Boston, MA, USA.

${ }^{9}$ University of New Hampshire, Durham, $\mathrm{NH}$, USA.

Received: 3 March 2020 Accepted: 17 September 2020

Published online: 30 September 2020

\section{References}

1. Drapalski AL, Milford J, Goldberg RW, Brown CH, Dixon LB. Perceived barriers to medical care and mental health care among veterans with serious mental illness. Psychiatr Serv. 2008;59(8):921-4.

2. Young H, Miyamoto S, Ward D, Dharmar M, Tang-Feldman Y, Berglund L. Sustained effects of a nurse coaching intervention via telehealth to improve health behavior change in diabetes. Telemed J E Health. 2014; 20(9):828-34.

3. Doarn CR. Telemedicine and psychiatry-a natural match. mHealth. 2018;4: 60-0.

4. Batsis JA, McClure AC, Weintraub AB, et al. Feasibility and acceptability of a rural, pragmatic, telemedicine-delivered healthy lifestyle programme. Obes Sci Pract. 2019;5(6):521-30.

5. King DK, Shoup JA, Raebel MA, et al. Planning for implementation success using RE-AIM and CFIR frameworks: a qualitative study. Front Public Health. 2020;8:59.

6. Brunet N, Moore DT, Lendvai Wischik D, Mattocks KM, Rosen MI. Increasing buprenorphine access for veterans with opioid use disorder in rural clinics using telemedicine. Subst Abus. 2020:1-8.

7. Serhal E, Arena A, Sockalingam S, Mohri L, Crawford A. Adapting the consolidated framework for implementation research to create organizational readiness and implementation tools for project ECHO. J Contin Educ Heal Prof. 2018;38(2):145-51.

8. Stevenson L, Ball S, Haverhals LM, Aron DC, Lowery J. Evaluation of a national telemedicine initiative in the veterans health administration: factors associated with successful implementation. J Telemed Telecare. 2018;24(3): 168-78.

9. Government US. Census Bureau statistics. 2012. Accessed 20 Jan 2013:2013.

10. Jensen MD, Ryan DH, Apovian CM, et al. 2013 AHA/ACC/TOS guideline for the management of overweight and obesity in adults. Circulation. 2014; 129(25 suppl 2):S102-38.

11. Diabetes Prevention Program Research G. The diabetes prevention program (DPP): description of lifestyle intervention. Diabetes Care. 2002;25(12):216571.

12. Pinnock H, Barwick M, Carpenter $\mathrm{CR}$, et al. Standards for reporting implementation studies (StaRI): explanation and elaboration document. BMJ Open. 2017;7(4):e013318.

13. Damschroder LJ, Aron DC, Keith RE, Kirsh SR, Alexander JA, Lowery JC. Fostering implementation of health services research findings into practice: a consolidated framework for advancing implementation science. Implement Sci. 2009;4:50

14. Kahwati LC, Lance TX, Jones KR, Kinsinger LS. RE-AlM evaluation of the veterans health Administration's MOVE! Weight management program. Transl Behav Med. 2011;1(4):551-60.

15. Fernandez ME, Walker TJ, Weiner BJ, et al. Developing measures to assess constructs from the inner setting domain of the consolidated framework for implementation research. Implement Sci. 2018;13(1):52.

16. Bond GR, Drake RE, Rapp CA, McHugo GJ, Xie H. Individualization and quality improvement: two new scales to complement measurement of program fidelity. Admin Pol Ment Health. 2009;36(5):349-57.
17. Haug NA, Shopshire M, Tajima B, Gruber V, Guydish J. Adoption of evidence-based practices among substance abuse treatment providers. J Drug Educ. 2008;38(2):181-92.

18. Boyatzis RE. Transforming qualitative information: thematic analysis and code development. Thousand Oaks, London \& New Delhi: SAGE Publications; 1998.

19. Bahadori M, Teymourzadeh E, Ravangard R, Raadabadi M. Factors affecting the overcrowding in outpatient healthcare. J Educ Health Promotion. 2017;6.

20. Brown JD, Hales S, Evans TE, et al. Description, utilisation and results from a telehealth primary care weight management intervention for adults with obesity in South Carolina. J Telemed Telecare. 2018. https://doi.org/10.1177/ $1357633 \times 18789562$.

21. Ceccarini M, Borrello M, Pietrabissa G, Manzoni GM, Castelnuovo G. Assessing motivation and readiness to change for weight management and control: an in-depth evaluation of three sets of instruments. Front Psychol. 2015;6:511.

22. Damschroder LJ, Goodrich DE, Robinson CH, Fletcher CE, Lowery JC. A systematic exploration of differences in contextual factors related to implementing the MOVE! Weight management program in VA: a mixed methods study. BMC Health Serv Res. 2011;11:248.

23. Kinsinger $L S$, Jones KR, Kahwati $L$, et al. Design and dissemination of the MOVE! Weight-management program for veterans. Prev Chronic Dis. 2009; 6(3):A98.

24. Rutledge T, Skoyen JA, Wiese JA, Ober KM, Woods GN. A comparison of MOVE! Versus TeleMOVE programs for weight loss in veterans with obesity. Obes Res Clin Pract. 2017;11(3):344-51.

25. Barnason S, Zimmerman L, Schulz P, Pullen C, Schuelke S. Weight management telehealth intervention for overweight and obese rural cardiac rehabilitation participants: a randomized trial. J Clin Nurs. 2019.

26. Skoyen JA, Rutledge T, Wiese JA, Woods GN. Evaluation of TeleMOVE: a telehealth weight reduction intervention for veterans with obesity. Ann Behav Med. 2015;49(4):628-33.

27. Norton WE, McCannon CJ, Schall MW, Mittman BS. A stakeholder-driven agenda for advancing the science and practice of scale-up and spread in health. Implement Sci. 2012;7:118.

28. Al-Shahi Salman R, Beller E, Kagan J, et al. Increasing value and reducing waste in biomedical research regulation and management. Lancet. 2014; 383(9912):176-85.

29. Glasziou P, Altman DG, Bossuyt P, et al. Reducing waste from incomplete or unusable reports of biomedical research. Lancet. 2014;383(9913):267-76.

30. Ioannidis JP, Greenland S, Hlatky MA, et al. Increasing value and reducing waste in research design, conduct, and analysis. Lancet. 2014;383(9912):166-75.

\section{Publisher's Note}

Springer Nature remains neutral with regard to jurisdictional claims in published maps and institutional affiliations.

\section{Ready to submit your research? Choose BMC and benefit from:}

- fast, convenient online submission

- thorough peer review by experienced researchers in your field

- rapid publication on acceptance

- support for research data, including large and complex data types

- gold Open Access which fosters wider collaboration and increased citations

- maximum visibility for your research: over $100 \mathrm{M}$ website views per year

At $\mathrm{BMC}$, research is always in progress.

Learn more biomedcentral.com/submissions 